\title{
Lokalressourcen oder Zentraltheologie? Zur Relevanz und Situierung geographisch strukturierter Mythologie im Alten Ägypten
}

\author{
Joachim Friedrich QUACK
}

Der Mythos im Alten Ägypten ist bekanntlich kein ganz einfaches Phänomen. Eine bis heute sehr wirkmächtige Forschungstradition hat von seiner "Verborgenheit" gesprochen und ihn für das Nilland als dezidiert spätes Phänomen angesehen. ${ }^{1}$ Ich selbst sehe diese Fragen durchaus etwas anders und würde die Frage lieber darauf verlagert wissen wollen, in welchen Situationen die Benutzung des Mythos in welchem Medium opportun war. ${ }^{2}$

Heutzutage ist man aus der Außenperspektive geneigt, die Mythen einer Kultur als homogenen Block zu betrachten; und so verpackt kann man sie dann in populären Büchern als Übersetzungssammlung oder lose Nacherzählung kaufen. Unausgesprochene Annahme ist, daß es eine einheitliche, landesweit anerkannte Theologie gibt; d.h. „der alte Ägypter" hätte das, was man so deklariert, insgesamt als Teil seiner Tradition aufgefaßt und allenfalls noch um Episoden ergänzt, die uns aufgrund von Überlieferungslücken nicht bekannt sind. Nun ist aber „der alte Ägypter" als solcher ein Konzept, mit dem ich nie sonderlich glücklich geworden bin. Es gab mehrere Millionen Einwohner eines Landes von fast Iooo Kilometer Länge, und diese auch noch in sozial höchst unterschiedlichen Situationen. Es ist nicht ohne Anmaßung, für diese doch zahlenstarke und in sich uneinheitliche Gruppe eine einzige übergreifende Tradition religiösen Wissens zu postulieren. Immerhin ist in der Forschung für andere Kulturen, insbesondere Indien, schon sehr bewußt ein Unterschied zwischen "großen“, also landesübergreifenden, und „kleinen“, also lokalen Traditionen gemacht worden. ${ }^{3}$ Sicher ist Ägypten in sich weniger disparat als Indien. Es fehlt schon allein alles, was dem dort als „Sanskritisierung" bezeichneten Vorgang entsprechen könnte, einfach weil die Sprachen-

I J. Assmann, „Die Verborgenheit des Mythus im Alten Ägypten“, Göttinger Miszellen 25 (1977) 7-43.

2 J.F. Quack, „Erzählen als Preisen. Vom Astartepapyrus zu den koptischen Märtyrerlegenden", in: H. Roeder (Hg.), Erzählen in frühen Hochkulturen (im Druck).

3 Immer noch eine Standarduntersuchung ist McKim Marriot, „Little Communities in an Indigenous Civilization", in: McKim Marriot (ed.), Village India. Studies in the Little Community (1955) I7I-222. 
situation eine andere ist. Dennoch kann man einmal den Versuch machen, nach ortsspezifischen religiösen Traditionen zu suchen, zumal es durchaus Quellen gibt, welche wenigstens für die späten Epochen Ägyptens, also ab dem I. Jahrtausend v. Chr., einem solchen Unternehmen Aussicht auf Erfolg geben.

Eine ganz spezielle Textgattung, die ohnehin wenig zum Konzept eines angeblich mythenscheuen Ägypten paßt, sind nämlich große textliche Zusammenstellungen von Mythen in geographischer Organisation. Bei diesen wiederum sollte man zwei Basistypen unterscheiden. Zum einen gibt es die übergreifenden Sammlungen, welche für ganz Ägypten oder zumindest für wesentliche Teile davon die relevanten Mythen und religiösen Traditionen zusammenstellen. Musterbeispiele hierfür sind erst rezent zugänglich gemacht worden, besonders zum einen das Mythologische Handbuch, von dem Jürgen Osing inzwischen eine Florentiner Handschrift der Römerzeit publiziert hat, ${ }^{4}$ während weitere noch in Bearbeitung sind, zum anderen ein Handbuch der Mythologie des Deltas, das Dimitri Meeks jüngst nach einer frühsaitischen Handschrift in Brooklyn vorgelegt hat. ${ }^{5}$ Angesichts der fragmentarischen Erhaltung der Handschrift wäre es dabei übrigens nicht auszuschließen, daß sie ursprünglich entweder ganz Ägypten abgedeckt hat oder daß Oberägypten auf einer weiteren, heute verlorenen Rolle behandelt wurde - jedenfalls wäre eine Beschränkung allein auf das Delta nicht wirklich ersichtlich, und an einzelnen Stellen greift der Text sogar evident oberägyptische Traditionen um Oxyrhynchos auf.

Neben diesen übergreifenden Darstellungen gibt es als weitere Kategorie die Textsorte, die seit Adolphe Gutbub gerne als „Monographien “ bezeichnet wird. ${ }^{6}$ Sie beschränken sich auf das Territorium eines einzigen Gaues, stellen dessen religiöse Traditionen aber in größerer Ausführlichkeit dar, als es in den oft änigmatisch knapp anmutenden übergreifenden Handbüchern möglich wäre. Vielleicht das bestbekannte Beispiel ist der Papyrus Jumilhac, eine fast neun Meter lange Papyrusrolle, in welcher der I8. oberägyptische Gau sowohl für seinen Hauptort als auch (viel kürzer) für die wichtigsten weiteren Orte abgehandelt wird. ${ }^{7}$

Während es hier nur eine einzige Handschrift gibt, ist das Buch vom Fayum in einer ganzen Reihe von Kopien auf uns gekommen, sowohl in hieroglyphischen und bebilderten Fassungen als auch in rein hieratischen Versionen ohne Bilder, und schließlich (derzeit noch unpubliziert) in einer hieratischen Version mit

4 J. Osing - G. Rosati, Papiri geroglifici e ieratici da Tebtynis (1998) I29-I 88.

5 D. Meeks, Mythes et légendes du Delta d'après le papyrus Brooklyn 47.218.84. Mémoires de l'Institut français d'archéologie orientale du Caire I 25 (2006).

6 A. Gutbub, Textes fondamentaux de la théologie de Kom Ombo. Bibliothèque d'étude, Institut français d'archéologie orientale, Kairo 47 (1973).

7 J. Vandier, Le papyrus Jumilhac (1961). 
demotischer Übersetzung und Kommentierung. ${ }^{8}$ Quasi als Papyrus nennen kann man auch das vieldiskutierte „Denkmal memphitischer Theologie“, das sich selbst als Abschrift von einem Papyrus ausgibt und auch wenig Anlaß zu Zweifeln an dieser Version liefert. ${ }^{9}$ Diesen Text möchte ich als Monographie des memphitischen Raumes interpretieren, was hoffentlich endlich für ein angemesseneres Verständnis und einen besseren Zugang sorgen wird.

Diese Textgattung gibt es sowohl auf Papyrus als auch monumentalisiert in den Tempeln, wobei Papyri der Intention nach eine Gesamtschau bieten, während die Steininschriften meist evident abkürzen, d.h. nur einen Einzelteil abbilden. Musterfälle sind hier Inschriften z. B. in den Krypten von Dendara (Dendara VI, I55, I5 - I59, 2 und 165, 10 - 169,7) ${ }^{10}$ oder in den Tempeln von Kom Ombo, ${ }^{11}$ Tôd $^{12}$

8 Edition H. Beinlich, Das Buch vom Fayum. Zum religiösen Eigenverständnis einer ägyptischen Landschaft. Ägyptologische Abhandlungen 5I (199I); ergänzend H. Beinlich, "Ein Fragment des Buches vom Fayum (W/P) in Berlin“, Zeitschrift für ägyptische Sprache und Altertumskunde I 23 (1996) IO-I 8; ders., „Hieratische Fragmente des ,Buches vom Fayum' und ein Nachtrag zu BF Carlsberg", Zeitschrift für ägyptische Sprache und Altertumskunde I 24 (I997) I-22; ders., „Drei weitere hieratische Fragmente des „Buch vom Fayum “ und Überlegungen zur Meßbarkeit der Unterwelt“, Zeitschrift für ägyptische Sprache und Altertumskunde 126 (I999) I-I 8, Taf. I-4. Kalkulation der derzeit faßbaren Menge wirklich verschiedener Handschriften bei J.F. Quack, „Die Dienstanweisung des Oberlehrers im Buch vom Tempel“, in: H. Beinlich - J. Hallof - H. Hussy - Chr. von Pfeil (Hgg.), s. Ägyptologische Tempeltagung Würzburg, 23.-26. September 1999. Ägypten und Altes Testament 33/3 (2002) I 59-171, dort S. I66 Anm. 25; eine etwas niedrigere Zahl setzt K. Ryholt, „On the Contents and Nature of the Tebtunis Temple Library. A Status Report", in: S. Lippert - M. Schentuleit (Hgg.), Tebtynis und Soknopaiou Nesos. Leben im römerzeitlichen Fajum (2005) I 4I-I70, dort I48f. mit Anm. 33 an.

9 Wichtigste Texteditionen und Bemerkungen bei K. Sethe, Dramatische Texte zu altägyptischen Mysterienspielen. Untersuchungen zur Geschichte und Altertumskunde Ägyptens 10 (1928) I-80; H. Junker, Die Götterlehre von Memphis (Schabaka-Inschrift). Abhandlungen der Preußischen Akademie der Wissenschaften 1939/23 (1940); ders., Die politische Lehre von Memphis. Abhandlungen der Preußischen Akademie der Wissenschaften 194I/6 (194I); J. P. Allen, Genesis in Egypt. The Philosophy of Ancient Egyptian Creation Accounts. Yale Egyptological Studies 2 (1988) 42-46 und 91-93; rezente Neubearbeitung durch A. El Hawary, Schöpfung als die letzte (Be)Gründung. Die Memphitische Theologie und die Siegesstele des Pije - zwei Zeugen kultureller Repräsentation in der 25. Dynastie. Orbis biblicus et orientalis (in Vorbereitung). Zur Datierung und zur Frage der Echtheit der Fundangabe vgl. A. von Lieven, Grundriß des Laufes der Sterne. Das sogenannte Nutbuch, The Carlsberg Papyri 8. The Carsten Niebuhr Institute of Near Eastern Studies, Publications 3I (2007) 255-257.

Io Diese Texte sind stark auf die Kultnamen der Orte fixiert und enthalten wenig narrative mythische Entwicklungen.

I I Diese bei Gutbub, Textes fondamentaux, a. O. (Anm. 6) behandelt, einschließlich einiger sicher nicht zur selben Gattung gehöriger Texte.

I 2 Ediert in É. Drioton - G. Posener - J. Vandier - J. C. Grenier, Tôd. Les inscriptions du temple ptolémaïque et romain I. La salle hypostyle, textes $N^{o s} I-I 72$. Fouilles de l'Institut français d'archéologie orientale de Cairo I8/I (I980); Chr. Thiers, Tôd. Les inscriptions du temple 
oder Esna, ${ }^{13}$ in denen bestimmte mythische Episoden erzählt werden. Sehr gerne finden sich diese Monographien gerade in Durchgangssituationen, wo die verfügbaren Flächen des Steines groß angelegte Bildkompositionen schwierig machen, eine textlastige Ausschmückung jedoch dem Auge des Durchschreitenden eine rasche und gründliche Orientierung bieten konnte. Weiterhin finden sich Monographien gerne als Dekoration später Naoi mit Kultstatuen von Gottheiten. ${ }^{14}$

Allerdings dürfte es sinnvoll sein, gerade diese Darlegung etwas zu nuancieren. Der Vorgang mag insofern korrekt beschrieben sein, als die Monumentalversionen mutmaßlich tatsächlich von Papyrushandschriften abhängig sind, die als Vorlagen benutzt wurden; und in der modernen Forschung ist sogar evident, daß es etwa Gutbub darum gegangen ist, quasi den Papyrus Jumilhac von Kom Ombo zu rekonstruieren. Jedoch kann man diese Papyrushandschriften nur mit großen Einschränkungen als homogene Kompositionen bezeichnen. $\mathrm{Zu}$ offensichtlich ist es, daß sie erst langsam gewachsen sind und auf Einzelpassagen höchst unterschiedlichen Alters zurückgreifen. Ich selbst habe dies vor kurzem für den Papyrus Jumilhac untersucht, wo man mehrere verschiedene Hauptstufen der Redaktion im Neuen Reich mit Weiterarbeit bis in die Spätzeit fassen kann. ${ }^{15}$ Für das Buch vom Fayum oder das Handbuch des Deltas wäre diese Arbeit noch zu leisten, könnte aber ohne weiteres durchgeführt werden. ${ }^{16}$ Dabei hat man teilweise so eklatante Phänomene zur Hand wie die Tatsache, daß das Handbuch des Deltas in einer Sektion über Heliopolis einige Sätze aus dem epigraphisch seit der 19. Dynastie belegten (aber sicher noch deutlich älteren) Grundriß des Laufes der Sterne aufgreift, diese aber in ihrer Reihenfolge erheblich modifiziert und lexikalisch gelegentlich modernisiert. ${ }^{17}$

ptolémä̈que et romain II. Textes et scènes $n^{\text {os }} 173-329$. Fouilles de l'Institut français d'archéologie orientale de Cairo I8/2 (2003).

I3 Zu Esna vgl. besonders H. Sternberg, Mythische Motive und Mythenbildung in den ägyptischen Tempeln und Papyri der griechisch-römischen Zeit. Göttinger Orientforschungen $\mathrm{IV} / \mathrm{I} 45$ (1985), bes. S. 36-109.

I4 Vgl. etwa V. Rondot, „Une monographie bubastide“, Bulletin de l'Institut français d'archéologie orientale 89 (1989) 249-270; ders., "Le naos de Domitian, Toutou et les sept flèches“, Bulletin de l'Institut français d'archéologie orientale 90 (I990) 303-338; G. Goyon, „Les travaux de Chou et les tribulations de Geb d'après le Naos 2248 d'Ismailia“, Kêmi 6 (1936) I42; Chr. Leitz, Altägyptische Sternuhren. Orientalia Lovaniensia Periodica 62 (1995) 3-50.

I5 J. F. Quack, „Corpus oder Membra disjecta. Zur Sprach- und Redaktionskritik des Papyrus Jumilhac“, in: W. Waitkus (Hg.), Diener des Horus. Festschrift für Dieter Kurth zum 65. Geburtstag. Aegyptiaca Hamburgensia I (2008) 203-228.

I6 Erinnert sei hier etwa daran, daß die Sektion über die Grenzen Ägyptens im Buch vom Fayum (Ed. Beinlich Z. 91 5-942) in der Wahl der Präpositionen $n-\check{c} 3 i$, "von“ und $r-h n-r$ „bis" deutlich demotische Spracheinflüsse zeigt.

17 von Lieven, Grundriß des Laufes der Sterne, a. O. (Anm. 9) 455-463. 
Als erstes konkretes Fallbeispiel greife ich einen Text aus dem Brooklyner mythologischen Handbuch auf, der unter den Titel „Osiris als Geflügelpastete in Letopolis" gestellt werden könnte. Der Text lautet im Gesamtzusammenhang der Sektion:

„Betreffend das Darreichen der Stäbe: Betreffend den Feiertag, der in Letopolis begangen wird, so heißt er ,Darreichen der Stäbe $e^{`}$. Betreffend(?) ${ }^{18}$ die beiden Herren, und ebenso(?) ihren Kampf, so war es so, daß es zu einem Heldenwerk überging, wobei sie es gemeinsam als Werk wiederholten beim Vogelfang, als die Seelen seiner Rebellen aufstiegen. Er fing die Seele des Müdherzigen ein, wobei es so war, daß sie <als> fähige Seelen aufstiegen, wobei es so war, daß er als sein Vater herabschwebte, wobei es so war, daß er ihn heftigst schlug. Sein Vater litt, ohne daß er es wußte.

Dann bereitete Horus sein Klappnetz vor, um die Seelen - Variante: Rebellen - einzufangen in Form von (? $)^{19}$ Amsti, Hapi, Duamutef und Kebehsenuef, als sie herabschwebten als das, was auffliegt und landet, auf dem Sand des Landes von Letopolis. Die Seele des Müdherzigen ließ sich bei ihnen nieder. Da ließ Horus seinen Strick zur Erde nieder, um die Seelen einzufangen, um seine Feinde zurückzutreiben. Nun kamen die Seelen dieser Götter, indem sie sich bei seinem Fangnetz niederließen. Es, war fern davon', daß man die Seele seines Vaters schlug. Er verstarb nicht sofort. Da strengte sich Thot gemeinsam mit Horus dabei an, ihn vielfach zu schlagen.

Dann ließ Thot ihn in Stoff einwickeln. Man grenzte ihn ab mit der zugehörigen Behandlung im Goldhaus. Seine Einwicklung wurde gemacht, als er aus ihm herauskam. Dann legte er ihn in einen Sarkophag auf dem Feld. Er ist dort bis heute als Serech (Palastfassade) des Herrn von Letopolis. Schentait und Meherchetes grenzen von ihm ab, während sein Sohn Horus von ihm fernhält. Da waren die Gottessubstanzen mit ihm genau so" (pBrooklyn 47.218.84, 8, 2-II).

Das ist zunächst einmal ein Text, der inhaltlich evident inhomogen und nicht aus einem, ja wahrscheinlich noch nicht einmal aus zwei Güssen ist. Zu deutlich wird, wie er Textbrocken zusammenstoppelt und mehrfach auf dasselbe Thema zu sprechen kommt. Gleichzeitig ist es inhaltlich ein ziemlich unerhörter Text. Zwar kennen wir auch sonst vage Anzeichen dafür, daß Thot sich Osiris gegenüber nicht immer positiv verhalten hat. ${ }^{20} \mathrm{Daß}$ er und vor allem Horus selbst sich des aktiven Totschlags an der Seele des Osiris schuldig gemacht haben, ist jedoch kaum normaler Standard. Selbst wenn man hier das Ganze als Versehen darstellt, bei dem der Ba des Osiris nicht als solcher erkannt wurde, bleibt Horus als derjenige, der seinen Vater tötet statt ihn zu beschützen, doch eine schockierende Gestalt. Die Brutalität der Behandlung des mit vielen kraftvollen Hieben geradezu zu Mus gemachten Gottes kommt hinzu. Man denkt zuerst an eine ganz obskure

I8 Hier und an manchen weiteren Stellen der Handschrift (4, 3; I 4, IO. I I (bis); I 5, I 2 ) vermute ich, daß in eine (auch sonst bezeugte) spätzeitliche Orthographie für $i r$ darstellt.

I9 Ich würde die Präposition $m$ an dieser Stelle lieber so denn als „zusammen mit“ auffassen.

20 Vgl. G. Meurer, Die Feinde des Königs in den Pyramidentexten. Orbis biblicus et orientalis I 89 (2002) 59-63. 


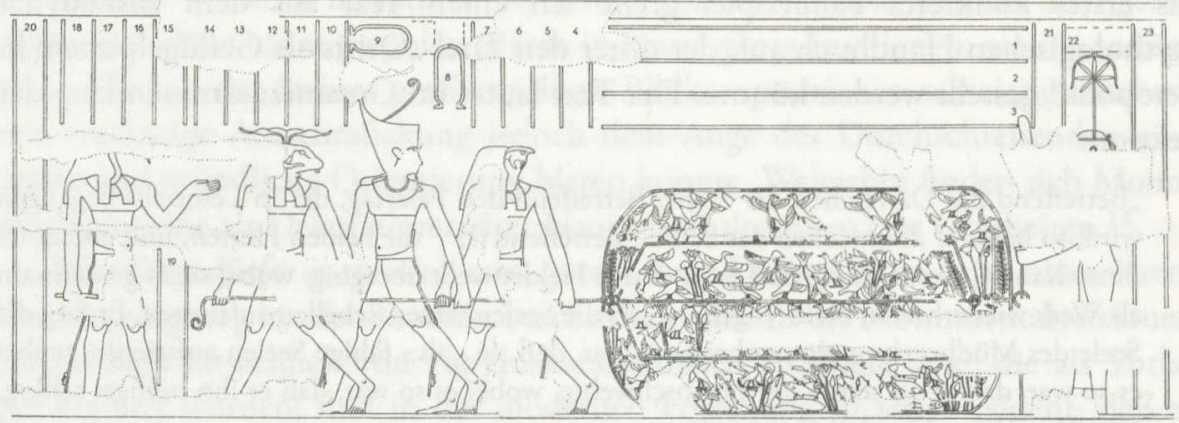

Abb. I: Götter unter Beteiligung des Horus beim Vogelfang unter Leitung durch Thot, Relief im Tempel von Esna.

lokal entwickelte Konzeption, wenn nicht gar an einen Versuch bewußter Mythenkorrektur. Tatsächlich läßt sich aber zeigen, daß die Situation doch etwas anders gelagert ist.

Sinnvollerweise sollte man die Kernepisode des Mythos zunächst im Rahmen sonstiger Ausdeutungen des Vogelfanges mit dem Klappnetz betrachten. Drei Tempelszenen, eine aus dem Neuen Reich in Karnak, zwei weitere in Edfu und Esna aus der griechisch-römischen Zeit, zeigen, wie dieses Bild als Einfangen und Bezwingen von Feinden und Rebellen verstanden wurde. ${ }^{21}$ Die beiden späten Versionen, insbesondere diejenige in Esna, geben auch explizit Letopolis als Schauplatz an, ${ }^{22}$ was als zusätzliches Zeichen für die feste lokale Fixierung bewertet werden kann (Abb. I).

Die eine Schicht unseres Textes, nämlich die Identifizierung der Vögel als Feindgestalten, läßt sich somit relativ unproblematisch erklären. Heikler ist es mit derjenigen, welche die Vögel zur Seele des Müdherzigen, also des Osiris, sowie meinem Verständnis nach auch zu den Horuskindern macht. Für letztere ist immerhin die Vogelgestalt in einer typischen Szene, nämlich dem Aussenden der vier Vögel in die Himmelsrichtungen, sehr gut belegt; dies wird im Minfest in Medinet Habu (Medinet Habu IV, 205, 14-27; 213, 5-17), in Edfu (Edfou V, 132, 10-133,4) und in den Osiriskapellen des Tempels von Dendera (Dendara X, 56, I-4 und 61,3-6; Taf. I6) dargestellt. ${ }^{23}$

2 I M. Alliot,„Les rites de chasse au filet dans les temples de Karnak, d'Edfou et d'Esna “, Revue d'Égyptologie 5 (1946) 57-II8; J.F. Quack, „Das Pavianshaar und die Taten des Thot", Studien zur Altägyptischen Kultur 23 (1996) 305-333, dort 3 I 9 f.

22 Meeks, Mythes et légendes, a. O. (Anm. 5) Io.

23 Vgl. etwa O. Keel, Vögel als Boten. Studien zu Ps 68, I2-I4, Gen 8, 6-I2, Koh I0, 20 und dem Aussenden von Botenvögeln in Ägypten. Orbis biblicus et orientalis I 4 (1977), bes. 103-I 42. 
Hier sollte auch die Bewertung des Vogelfanges im kürzlich veröffentlichten sogenannten Thotbuch zur Sprache kommen. ${ }^{24}$ In ihm werden diese Aktionen nämlich auf mehreren Ebenen ausgedeutet. Neben der simplen Option der Nahrungsbeschaffung und der nur leicht präsenten, daß es um Abwehr von Unheil geht, ist die wichtigste Komponente diejenige des Wissenserwerbes, bei dem die Vögel für Wissen stehen bzw. mit der Bezeichnung der fundamentalen Texte als „Seelen des Re“ korreliert werden - und Seelen haben nach ägyptischer Konzeption Vogelgestalt.

Gerade diese Konstellation bringt es mit sich, daß die Seelen bzw. Vögel, die gefangen werden, nicht mehr einfach die Seelen der Rebellen sind, sondern auch als die „vortrefflichen Seelen“ verstanden werden können, wie es der Deltapapyrus bereits als Alternative offeriert. Eine derart intellektuelle Ausdeutung wird sicher auch durch die Präsenz des Schreibergottes Thot befördert worden sein, der sehr typisch zur Vogelfangszene hinzugehört. Diese vortrefflichen Seelen sind aber wiederum in ägyptischen religiösen Texten typisch solche, welche Osiris folgen, so daß dessen Präsenz sich mit fast zwingender Logik ergibt.

Realer Hintergrund einer solchen Episode sollte zunächst einmal sein, daß im Bereich von Letopolis der Vogelfang eine besondere Rolle spielte. Vogelfang mit Klappnetzen, d. h. in ökonomisch relevanter Massenproduktion, ${ }^{25}$ ist ein Phänomen, das primär mit den großen Wanderungen zu tun hat, bei denen im Vogelzug große Schwärme gemeinsam eine Landschaft durchziehen, konkret also auf der Route von Europa nach Innerafrika. ${ }^{26}$ Hier wäre in Zusammenarbeit mit Geographen und Ornithologen zu überprüfen, inwieweit die Sand- und Wasserflächen um Letopolis für die Zugvögel auf Wanderschaft tatsächlich ein besonders geeignetes Rastareal bieten. Ohne dies heute bereits definitiv nachweisen zu können, würde ich es erwarten.

Zusammengenommen hat man also eine Lokaltradition, die an spezifisch lokalen landschaftlichen Gegebenheiten und Ressourcen hängt, konkret an geeigneten Vogelfanggründen. Daß eine vor Ort wirtschaftlich wichtige Tätigkeit sich auch im Mythos widerspiegelt, braucht uns nicht zu überraschen; und dieser Mythos kann eigentlich so nur vor Ort entwickelt sein. Zunächst wirkt das also nach

24 Edition R. Jasnow - K.-Th. Zauzich, The Ancient Egyptian Book of Thot. A Demotic Discourse on Knowledge and Pendant to the Classical Hermetica (2005); vgl. weiter J. F. Quack, „Die Initiation zum Schreiberberuf im Alten Ägypten“, Studien zur Altägyptischen Kultur 36 (2007) 249-295 (zum Vogelfang bes. 253 f.); ders., „Ein ägyptischer Dialog über die Schreibkunst und das arkane Wissen", Archiv für Religionsgeschichte 9 (2007) 259-294.

25 Vgl. O. Mahmoud, Die wirtschaftliche Bedeutung der Vögel im Alten Reich. Europäische Hochschulschriften, Reihe 38 Archäologie, Band 35 (I99I).

26 M. Herb, Der Wettkampf in den Sümpfen. Quellenkritische, naturkundliche und sporthistorische Untersuchungen zu einem altägyptischen Szenentyp. Nikephoros Beiheft I (200I) 346348 . 
einer lokalen kleinen Tradition. Andererseits ist unübersehbar, daß diese Lokalform schon in der Benennung der Protagonisten als Horus, Thot und Osiris keine ganz unabhängige Entwicklung darstellt, sondern die Kenntnis der landesweiten Grundstrukturen voraussetzt. Ebenso zeigt auch die Tatsache, daß landesweit das Thema „Vogelfang durch Götter" am Ort Letopolis festgemacht wird, daß es keineswegs auf dem Status einer kleinen Tradition geblieben, sondern Teil der großen geworden ist. Wesentliche Komponenten, insbesondere die changierende Bewertung der gefangenen Vögel, teils als negative, teils als positive Gestalten, ohne welche die Tragik der überlieferten Fassung gar nicht denkbar wäre, scheinen sogar eng an zentral konzipierten Texten zu hängen. Schließlich sollte man beachten, wie im Mythos letztlich eine Erklärung für einen konkreten Zug geliefert wird, der bis zum Zeitpunkt der Niederschrift des Papyrus in der Landschaft wahrzunehmen war, nämlich die Bestattung der Gottessubstanz im Acker von Letopolis.

Als zweites Beispiel möchte ich den "Speienden Hund von Assiut" anführen. Dieser Text ist im Mythologischen Handbuch aus Tebtynis überliefert. Um den Text richtig einzubetten, möchte ich ihn im größeren Zusammenhang zitieren. Es heißt dort:

„Betreffend Assiut (und) die (Göttin) von der Küche. Es ist ein geschützter Gau. Man bezeichnet das Bewachen $(s i w)$ der Dinge als ,Assiut' $\left(s 3 w . t t^{3}\right)$. Ein Hund ernährte sich davon und spie es aus. Man bezeichnet die Speisen in Einwickelung als, Djetef-chent'. Das ist das Götterbild in Assiut, das man in der Küche der Herrin der 16 aufsucht, bis zum Tag des Dastehens. ${ }^{27}$ Betreffend ${ }^{28}$ Hämatit und Gold, so sind es die Knochen des Horus. Betreffend das Eisen ( $b i 3$-n-p.t), so ist es die Knochen des Seth. Sie kämpften einmal, so wie sie es seit vordem getan hatten. Upuaut verbarg das Zerstückelte in der Höhle seines Hauses. Horus verbarg ihn/sich und sprang wieder empor, um seinem Vater freie Bewegung zu geben. Er kontrollierte die Rotte des Finsterlings. Er leckte die Fäulnisstoffe (iw.tiw) der Mumie auf. Deswegen kam es, daß der Schakal (s3b), der über der Götterfigur (wrm) ist, hergestellt wird (?). ${ }^{29} \mathrm{Er}$ spie aus, was er verschluckt hatte. Die edle Gestalt wurde festgesetzt. Die Ausflüsse des Gottes wurden bewacht. Er [...] die Substanz seines Vaters Osiris. Man nennt ihn: ,Dieser Hund hat gefressen und ausgespieen und sich umgewandt, um es wieder zu fressen', als man ,Hund' (iwiw) sagte, als er kam (iwi), um zu essen, was er

27 Der "Tag des Dastehens“ (hrw $n^{c} h^{\top}$ ) wird im Buch vom Tempel als Termin der Amtsübergabe vom Vater auf den Sohn genannt, vgl. J.F. Quack, „Ämtererblichkeit und Abstammungsvorschriften bei Priestern nach dem Buch vom Tempel“, in: M. Fitzenreiter (Hg.), Genealogie - Realität und Fiktion von Identität. Internetbeiträge zur Ägyptologie und Sudanarchäologie V (2005) 97-102, dort 98.

28 Ungeachtet Osings Lesung für den unpublizierten Paralleltext kann aus inhaltlichen Erwägungen $\mathrm{m}$. E. nichts anderes als $i r$ angesetzt werden.

29 Ich möchte im Zeilenübergang [ms]s. $(t) w s 33 b$ pw hri wrm.t $h r=s$ lesen; Osings Lesung $s i w$ am Zeilenbeginn ist weder paläographisch evident noch sprachlich sinnvoll analysierbar. 
ausgespieen hatte, wobei er zu(?) seinen Herren kam(?), ${ }^{30}$ wobei er vor ihm bellte für seine Belohnung. Sein Herr wurde matt. Er fraß nicht und haßte sehr, was er verschlungen hatte; die Ausflüsse, die aus dem Abbild gekommen waren sowie den Fingern des Müdherzigen. Dann spie er sie zu Boden aus. Das ist ein Geben, das er machte, nachdem er sie erneut verzehrt hatte" (PSI Inv. I 72, $\mathrm{x}+3, \mathrm{I}-\mathrm{I} 2)$.

Typisch für den ägyptischen Mythos sind die „Wortspiele“, ${ }^{31}$ mit denen über das Instrumentarium des ähnlichen Klanges inhaltliche Stimmigkeit erzielt wird, hier zwischen „Hund“, „kommen“ und „Fäulnisstoffe“. Auch dies ist ein inhaltlich nicht ganz unproblematischer Text, bedenkt man den Umgang des Hundes mit Osiris. Ihn zu bewachen ist lobenswert und das, was man von einem guten Wachhund erwartet. Auch das erwartungsfrohe Bellen des Hundes, der von seinem Herrn eine Belohnung mit Futter erwartet, scheint braves Verhalten zu sein. Manche Hunde sollen auch eine Vorliebe dafür haben, ihre Herrchen oder sonst beliebte Personen abzuschlecken. Aber richtig Substanz vom Herrn abzulecken, dann auszuspeien und erneut zu verschlingen, zeigt nicht nur schlechte Tafelmanieren, sondern auch einen arg respektlosen Umgang mit dem verstorbenen Gott. Gemildert wird die Sache allenfalls durch die psychologische Ausdeutung, dem Hund sei der Appetit vergangen, als er merkte, daß er von seinem Herren gefressen hatte, und deshalb habe er es wieder ausgespieen.

In dieser Passage dürfen wir zunächst einmal die Ätiologie für den Namen der Stadt Assiut (wörtlich „Wächter") erkennen, wobei der dortige Canidenkult prominent aufgearbeitet wird. Ein in seinem Umkreis hergestelltes Kultbild wird genauer erörtert. Für das Verhalten des Hundes kann man durchaus naturkundliche Parallelen anführen. So wenig dies den meisten heutigen Hundefreunden gefällt, aber Hunde brauchen für ihre Gesundheit ein gewisses Maß an bereits

30 Osings Lesung $h r$ iç̣t überzeugt mich weder paläographisch noch inhaltlich. Ich lese und verstehe $m$ als Schreibung für $n$.

3 I Der Begriff ist natürlich wenig angemessen, da es sich gerade nicht um ein Spiel im „entspannten Bereich" handelt, sondern um innerkulturell höchst relevante hermeneutische Prozesse. Vgl. zu ägyptischen Wortspielen einstweilen C. E. Sander-Hansen, „Die phonetischen Wortspiele des ältesten Ägyptischen“, Acta Orientalia 20 (I948) I-22; S. Morenz, „Wortspiele in Ägypten“, in: Festschrift Johannes Jahn zum XXII. November MCMLVII (1957) 23-32; W. Guglielmi, "Zu einigen literarischen Funktionen des Wortspiels“, in: Studien zu Sprache und Religion Ägyptens zu Ehren von Wolfhard Westendorf (1984) 49I506; F. Junge, „Zur ,Sprachwissenschaft' der Ägypter“, in: Studien zu Sprache und Religion Ägytens zu Ehren von Wolfhard Westendorf(1984) 257-272; A. Loprieno, „Puns and Word Play in Ancient Egyptian“, in: S. B. Noegel (ed.), Puns and Pundits. Wordplay in the Hebrew Bible and Ancient Near Eastern Literature (2000) 3-20; S. B. Noegel, „On Puns and Divination: Egyptian Dream Exegesis from a Comparative Perspective“, in: K. Szpakowska (ed.), Through a Glass Darkly. Magic, Divination \& Prophecy in Ancient Egypt (2006) 95-II9; S. B. Noegel - K. Szpakowska, ", Word Play' in the Ramesside Dream Manual“, Studien zur Altägyptischen Kultur 35 (2006) I93-2I 2. 
verfaultem Fleisch. Als reales Verhalten kann man zumindest beobachten, daß sie Futter erst einmal im Mund wegtragen, dann ausspucken, vergraben, und erst im richtigen Reifegrad wieder ausbuddeln und verzehren. Gerade der schockierendste Zug von allen, daß nämlich der Hund vom Leichnam und den Fäulnisprodukten des Osiris gefressen hat, ergibt sich einfach mit fast zwingender Logik daraus, daß Caniden eben eine gewisse Vorliebe für verfaultes Fleisch haben, Osiris aber eben der faulende Leichnam par excellence ist. ${ }^{32}$

Relevant ist hier auch, inwieweit die mittelägyptischen Regionen gerade um Assiut tatsächlich in besonderem Maße ein Lebensraum für bestimmte Caniden sind. Leider dürfte angesichts der völligen Umstrukturierung der Kulturlandschaft im Zusammenhang mit der heutigen weit höheren Bevölkerungszahl wenig Aussicht bestehen, für das Altertum zu verläßlichen Schlußfolgerungen zu kommen. Tatsächlich wird in rezenter Zeit über das Erscheinen eines als „Salawa" bezeichneten Caniden in der Region von Sohag/Luxor berichtet, bei dem es sich um eine Art Hund, aber mit kräftigerer Schnauze handeln soll. ${ }^{33}$ Jedoch mag es sich hier um eine „urban legend" handeln.

Demnach ist dieser Text also lokal basiert in dem Sinne, daß er sicher einen spezifischen Ortskult verarbeitet, mit einer gewissen Wahrscheinlichkeit auch ein Element der lokalen Fauna, und in gewissem Grad ein reales Verhalten eines Tieres. Es handelt sich aber auch nachweislich um eine überregional bekannte Episode, denn im "Geiertext des Thotbuches", auf den ich im Folgenden noch eingehen werde, wird gerade zum Gau von Assiut das Motiv des Speiens wieder aufgegriffen. ${ }^{34}$

Nun ist natürlich das Verhalten eines Caniden gegenüber einem verrottenden Leichnam irgendwie zu erwarten, und von daher überrascht es nicht, daß eine ähnliche Episode auch im Papyrus Jumilhac zu finden ist, nämlich im Zusammenhang einer Klassifizierung der verschiedenen Canidensorten (I5, 9-I6, 22) ${ }^{35}$ Diese zeichnet sich zunächst durch eine Fluktuation zwischen positiver und negativer Bewertung ein und desselben Tieres aus, die einen modernen Leser fast zum Wahnsinn treiben kann. Man würde sie a priori auf das Konto redaktioneller Schichten schieben wollen, aber zumindest in sprachlicher Hinsicht gibt es keine Indizien für eine Separierung der Einheiten. ${ }^{36}$ Hier heißt es dann auch von einem Hund:

32 Vgl. hierzu S. Banaschak - M. Grothoff, „Osiris - der grüne Totengott“, Göttinger Miszellen $\mathrm{I} 75$ (2000) I7-2I.

33 http://www.newton.cam.ac.uk/egypt/lxr96/Luxorg6.html.

34 Quack, „Die Initiation zum Schreiberberuf im Alten Ägypten“, a. O. (Anm. 24) 287.

35 Vgl. dazu A. von Lieven, „Das Göttliche in der Natur erkennen. Tiere, Pflanzen und Phänomene der unbelebten Natur als Manifestationen des Göttlichen“, Zeitschrift für ägyptische Sprache und Altertumskunde I3 I (2004) I 56-I72, dort S. I60-I62.

36 Quack, "Corpus oder membra disiecta", a. O. (Anm. I 5) $217 \mathrm{f}$. 
"Der ,Feind des ${ }^{`}$ Osiris ${ }^{37}$ war es, der bei ihm litt“ ( $15, \mathrm{I} 3 \mathrm{f}$.), von einem anderen, er habe in der Balsamierungsstätte an den Gliedern des Osiris geleckt und sich dabei in den Mumienbinden verheddert - was als Erklärung seiner teilweise weißen Fellfarbe dient (I6,3 f.). Das mythologische Motiv, daß ein Canide sich bei der Behandlung der Osirismumie vergißt und sie als Nahrung statt als schützenswertes Gut behandelt, ist also durchaus an verschiedenen Orten präsent.

Mein drittes Beispiel kann man unter die Überschrift „Isis als Ziel der Lust des Seth"stellen. Der Text steht im Rahmen der mythologischen Lokaltraditionen des Papyrus Jumilhac und lautet:

„Dann versammelte Seth seine Rotte erneut. Isis ging gegen ihn los, indem sie sich auf diesem Berg im Süden von Dun-Awi verbarg, und sie verwandelte sich in die Form ihrer Mutter Sachmet. ${ }^{38}$ Das Feuer kam gegen sie alle hervor; sie wurden verbrannt, sie wurden verzehrt von der Flamme. Man nennt sie, Hathor von den beiden Feuerbecken'. Dann erschuf sie sich dort einen Ort, um zu erkennen, was der Finsterling mit seiner Rotte machte. Man nennt ihn, Haus der Herrin der beiden Feuerbecken'. Betreffend den Priester dieser Göttin, ,Groß an Fressen ' ist sein Name. Dann sah Seth Isis an diesem Ort, und er verwandelte sich in einen Stier, der ihr nachlief. Sie veränderte ihre Gestalt in die einer Hündin mit einem Flintmesser an ihrem Schwanz. Sie lief vor ihm her, und er konnte sie nicht erreichen. Da ergoß er seinen Samen zu Boden. Darauf sagte diese Göttin: ,Es ist Abscheu, daß du Samen ergossen hast' (bw.t $m$ wt $t i=k k$ ) ). Da wuchs sein Same als Pflanze auf diesem Berg, und sein Name wurde $b t t-k 3$ (Flaschenkürbis?)." (pJumilhac 2, 2I-3, 5)

Nun mag der zentrale Punkt, der Samenerguß des Seth ohne Kopulationserfolg, etwas an die athenische Tradition über Athena und Hephaistos erinnern, dennoch möchte ich hier zunächst auf die lokalspezifischen Punkte fokussieren. Seth als Stier mag uns noch ganz vertraut sein, aber Isis als Hündin ist es schon erheblich weniger. Ebenso ist Sachmet als Mutter der Isis kaum ein Standardmodell. Man kann sogar guten Gewissens die Frage stellen, ob hier in einer früheren Entwicklungsphase vielleicht eine ganz andere lokale Göttin im Plot agierte, die erst später auf das Normmodell von Isis als erfolgreicher Widersacherin des Seth umgeschrieben wurde. Dafür spricht auch, daß die Göttin dieses Ortes auch als Hathor bezeichnet

37 Eine für Ägypten durchaus typische Ausdrucksweise; „Feind des NN“ wird verwendet, wenn einer kulturell positiv konnotierten Person Unheil zustößt. Vgl. G. Posener, „Sur l'emploi euphémique de $h f t j(w)$,ennemi(s)"“, Zeitschrift für ägyptische Sprache und Altertumskunde 96 (I970) 30-35; J. F. Borghouts, Lexikon der Ägyptologie III (I980), Sp. I 143 u. I 49 Anm. 94 s.v. „Magie“; J.F. Quack, „Sur l’emploi euphémique de $h f t$,ennemi` en démotique“, Revue d'Égyptologie 40 (1989) I97-198; ders., „Ein altägyptisches Sprachtabu“, Lingua Aegyptia 3 (I993) 59, 79, dort 6r Anm. 22 (mit weiterer Literatur); ders., „Rezension zu S. Lippert, Ein demotisches juristisches Lehrbuch“, Archiv für Papyrusforschung 5 I (2005) 173; G. Vittmann, Der demotische Papyrus Rylands 9. Ägypten und Altes Testament 38 (I998) $509 \mathrm{f}$.

38 So durch Zusatz eines Suffixes nachträglich aus Mut-Sachmet korrigiert. 
wird. Auf jeden Fall ist deutlich, wie hier der Mythos als Erklärungsmodell eine Sinnhaftigkeit der realen Gegebenheiten produziert: Ein Ort und sein Kult, dessen Namen Assoziationen zu Feuer und Verzehren liefern, wird in einem Mythos vom verzehrenden Feuer, das gegen Feindgestalten wirkt, plausibel gemacht - der konkrete Felstempel ist bis heute erhalten. ${ }^{39}$ Ein Vegetationszug, daß eine bestimmte Pflanzensorte in einer lokalen Region ein geeignetes Biotop gefunden hat, wird über den Mechanismus des Wortspiels mit deren Namen verkoppelt, und dieses Wortspiel produziert wiederum eine mythische Episode über unangemessenen Samenerguß.

Freie Auswahl des Textproduzenten ist natürlich, an welchem Götterpaar das dann konkret festgemacht wird. Daß man Seth als einen Partner wählt, ist vielleicht nicht sehr überraschend, spielt er doch im Mythos auch sonst die Rolle dessen mit übertriebener und ungeordneter Sexualität, der verschiedene Göttinnen vergewaltigt. ${ }^{40}$ Isis ist dagegen etwas weniger evident. Zwar kennen wir aus dem Streit zwischen Horus und Seth das Motiv, daß Isis in Verwandlung als schönes Mädchen ihre weiblichen Reize bei Seth spielen läßt. Aber eine aktive sexuelle Belästigung der Isis durch Seth kennt man sonst nicht einmal aus Texten wie einigen spätzeitlichen Zaubersprüchen, welche Isis sehr durch Seth bedrückt darstellen. ${ }^{41}$ Von den Fällen, die ich bislang vorgestellt habe, ist dies wohl derjenige, der am meisten lokal konzipiert wirkt und vielleicht in nicht viel mehr als den Namen der Hauptfiguren an die überregionalen Mythen angeschlossen ist. ${ }^{42}$ Es mag bezeichnend sein, daß der hauptsächliche Kultname der Göttin als „Hathor“, nicht Isis angegeben wird. Mir ist auch derzeit kein sicherer Beleg für eine Rezeption außerhalb des eng begrenzten regionalen Umfelds bekannt.

Im Papyrus Jumilhac kann man auch an einer anderen Stelle sehr gut nachverfolgen, wie ein ganz spezifischer Zug der Landschaft, nämlich die Anwesenheit eines Weinberges, eine Wurzel in der Mythologie erhält. Ohne den langen Text (13,15I4, 2I) in extenso zitieren zu können, seien die wesentlichsten Punkte resümiert. Ausgangspunkt sind zwei Kästen, in denen sich angeblich die Augen des Horus befunden haben. Sie werden zunächst von Seth geraubt und im Gebirge deponiert, wo er in Form eines Krokodils über sie wacht. Anubis verwandelt sich in eine große Schlange, um sie zu retten. Mit weiteren Schlangen in seinem Gefolge zerstört er

39 Vandier, Papyrus Jumilhac, a. O. (Anm. 7) so.

40 Meeks, Mythes et légendes, a. O. (Anm. 5) $255 \mathrm{f}$. Hier kann man auch an die Schilderung des ungezügelten Sexualverhaltens des sethianischen Menschen nach pChester Beatty III rt. $\mathrm{I}$, 7 f. $\mathrm{r} 3 \mathrm{f}$. denken.

4 I z. B. Metternichstele, Z. I73-175.

42 Vandier, Papyrus Jumilhac, a. O. (Anm. 7) rogf. leistet nicht viel mehr, als die Episode in etwas eigenen Worten nachzuerzählen. 
den Ort des Seth. Nunmehr gibt Anubis den beiden Kästen mit den Augen des Horus einen dauerhaften Ort. Als Isis kommt, um sie zu besichtigen, stellt man fest, daß sie in Weinstöcken ausgetrieben haben. Man baut eine Ansiedlung, wo sich Isis niederläßt und die Pflanzen begießt. Auf Bitte der Isis hin erhält Horus von Re dann auch Augen sowie das Recht auf den Thron seines Vaters Osiris. Letztlich wird damit die Existenz eines Weinberges und eines „Hauses der Gottesgemahlin“ als noch in der Aktualität des Papyrus präsenter Landschaftszüge erklärt, zudem nebenbei auch noch die Palme als Hypostase der Isis sowie die Präsenz einer Stele, die zum Ortsnamen Pa-Ahay, d.h. „die Stele“ führt.

Es fällt relativ leicht, die Realien zu erkennen, an denen der Mythos hier aufgehängt ist. Im lokalen Raum gibt es vermutlich einen Ruinenhügel und sicher eine Siedlung, die „Haus der Gottesgemahlin“ heißt, de facto vermutlich, weil sie von einer Gottesgemahlin des Amun bzw. für ihr Gut angelegt wurde. ${ }^{43}$ Daneben kann man die reale Existenz eines Weinberges plausibel ansetzen. Auch der Ortsname „die Stele“ ist sicher lokal in der Aktualität präsent gewesen. Über die Deutungsschiene des Weines als Horusauge wird aus diesen Realien nunmehr eine Anbindung an den Mythos etabliert. Die Konzeption als solche ist sicher lokal, da sie detailtopographische Realitäten aufgreift, um welche die Zentrale des Landes kaum mit gesteigertem Interesse gewußt haben wird. Die Grundparameter des dann kreierten Mythos bewegen sich jedoch in irgendwie vertrautem Rahmen - das Grundmuster des Streites zwischen Horus und Seth um das Erbe des Osiris und die Hilfe der Isis für Horus sind so präsent, daß man sich diesem Jargon kaum entziehen kann. Auch das Motiv, daß aus den vergrabenen Augen des Horus Pflanzen wachsen, ist sonst z.B. im Papyrus Chester Beatty I (Io, 3-5) bekannt. ${ }^{44}$ Lokalkonzeptionen können sich also der Zentraltradition kaum entziehen.

Andererseits muß man doch zur Ehrenrettung der lokalen Köpfe sagen, daß sie zumindest einige unkonventionelle Züge in die konkrete Ausformung des Mythos hineingebracht haben. Weder die Krokodilsform des Seth noch die Schlangenform des Anubis sind Fakten, auf die man im ersten Reflex sofort kommen würde.

Das Aufgreifen realer geographischer Landschaftszüge läßt sich auch sonst feststellen. Herausgegriffen sei hier eine kurze Notiz aus einer Abfolge von Einträgen, welche die verschiedenen Seen in der Region mit Namen und religiösen Hintergründen präsentiert. „Betreffend den See des Feuers, so ist er im Norden dieses Gaues. Anubis kämpfte an ihm mit Seth. Das Blut des Seth floß in ihn sowie

43 Der Ort ist bereits im pWilbour B I 7, I6 bezeugt, vgl. E. Graefe, Untersuchungen zur Verwaltung und Geschichte der Institution der Gottesgemahlin des Amun vom Beginn des Neuen Reiches bis zur Spätzeit. Ägyptologische Abhandlungen 37 (I98I) I05 $\$ 43$.

44 M. Broze, Mythe et roman en Egypte ancienne. Les aventures d'Horus et Seth dans le Papyrus Chester Beatty I. Orientalia Lovaniensia Analecta 76 (I996) 83 f. 
das Blut ,der Feinde' des Horus. ${ }^{45}$ Man nennt ihn See des Feuers bis zum heutigen Tag" (pJumilhac XI, I7f.). Einerseits kann man guten Gewissens annehmen, daß sowohl der Name "See des Feuers" als auch die mythologische Episode vom Fließen des Blutes auf der konkreten Erscheinung des Sees beruhen, dessen Wasser vielleicht infolge der Bodenbeschaffenheit oder eines Algenvorkommens relativ rötlich wirkte. Andererseits ist in der mythologischen Episode selbst ein eklatanter Bruch zu verzeichnen. Einerseits ist die eine Kampfpartei Anubis als Hauptgott des konkreten Gaues, andererseits wird Horus als derjenige bezeichnet, der konkret verletzt wird, also der überregionale Standardgegner des Seth. Ob hier eher eine zentrale Konzeption regionalisiert oder eine regionale an zentrale Normen angeglichen wurde, wird sich schwer absichern lassen, in jedem Fall ist die enge Verzahnung dieser beiden Bereiche unverkennbar.

Vielleicht am dezidiertesten auf die Landschaft bezogen sind Traditionen über Mineralvorkommen. Es ist auffällig, wie sehr die Ägypter eine Tendenz haben, die Präsenz farblich auffälliger Mineralien mythisch dadurch zu erklären, daß hier Körperflüssigkeiten ausgelaufen sind. Ein Beispiel ist etwa: „Dann kam die Rotte des Seth, um ihn zu suchen, indem sie sehr zahlreich waren. Sie lagerten auf diesem Berg im Süden von Dun-Awi. Dann ging Anubis in der Nacht gegen sie vor und richtete ein großes Gemetzel unter ihnen an. Er schlug ihre Köpfe mit einem $\mathrm{Mal}$ ab. Niemals war er dabei liebenswürdig(?). Ihr Blut floß dann auf diesem Berg. Deshalb entstand das Schesait-Mineral von Dun-Awi bis zum heutigen Tag" (pJumilhac II, I5-20; ähnlich auch XXII, IO-I2). Ein anderes Mineralvorkommen, nämlich das von Mennige(?) (oder Rötel?), wird ebenfalls durch Blut der Feinde produziert, die diesmal von einer Göttin in Skorpions- oder Schlangengestalt gestochen bzw. gebissen werden (pJumilhac III, IO-I2; XXIII, I2-I6).

Derartige Traditionen können nur lokal vor Ort aufgekommen sein, wo die Mineralvorkommen direkt vor Augen standen und nach einer Erklärung schrien. Gerade bei gelb-rötlichen Mineralien lag eine Verbindung mit Blut, das hier vergossen worden war, auf der Hand. Andererseits können sich die möglichen Konstellationen, unter denen dieses Blut konkret vergossen worden war, keineswegs beliebig entfalten, sondern werden an das Normmodell der Feindgestalt des Seth und seiner Anhängerschaft angehängt, denen wiederum Horus oder Isis als positive Helden gegenüberstehen.

Vergleichsweise origineller verfährt hier der Papyrus über die Mythologie des Deltas, der die Entstehung bestimmter Mineralien aus den Ausflüssen beim Verlöschen des Auges des Atum ( $7,9 \mathrm{f}$.) sowie den blutigen Ausflüssen der Horit $(9,2 \mathrm{f}$.) erklärt - beides Traditionen, für welche wirklich ähnliche Motivik in anderen Tex-

45 Vgl. für die euphemistische Wendung Anm. 37. 
ten kaum zu finden ist. ${ }^{46}$ Lokale Herkunft ist hier zweifelsfrei, auch wenn die großen Traditionen bei den Deutungsmustern fallweise behilflich sind.

Wie sehr die lokalen religiösen Denker umgekehrt kreativ auf die Tradition des Gesamtlandes zurückgreifen, zeigt sich auch in zwei strukturell hochinteressanten Sektionen im Rahmen des Buches vom Fayum. Die eine betrifft die verschiedenen Siedlungen im Gelände. Im Papyrus sind sie teilweise in den ersten Sektionen zu finden, die - geographisch gesprochen - den Bereich der Zuflüsse des Bahr Yussuf zum Fayumsee betreffen dürften, teilweise an den oberen und unteren Rändern eines Bereiches, der evident eben den See als Mitte der Zeichnung versteht. Sie werden relativ ausführlich thematisiert, wobei es zu jedem Ort einen kurzen „Paß“ gibt, welcher die wichtigste Information zusammenfaßt. Ein Beispiel ist etwa „Dieser Platz, Ideb (?) ist es. Der Tempel des Sobek von Krokodilopolis im südlichen See. Das ist Atum, der vor seinem See ist. Er ist an der südlichen Seite des Sees als Barriere zwischen Seth und Osiris. Imi-netjeri ist der Name des Priesters des Sobek, ${ }^{47}$ Herrn von Ideb. - Re, Herr von Ideb“ (Z. 22I-228). In der Nennung eines spezifischen Priestertitels geht dieser Eintrag bereits über das sonst übliche Formular hinaus. Relevant ist vor allem eine ganz essentielle Kenntnis der wichtigsten Elemente: Name des Ortes, ungefähre Lage und religiöse Zuordnung, d. h. Hauptgottheit. Ein wesentlicher Punkt kommt aber noch hinzu: Bei der religiösen Zuordnung wird über den Lokalgott Sobek hinaus der deutlich überregionale Atum bzw. Re genannt. Vor allem werden in vielen Fällen diese nichtregionalen Gottheiten spezifisch auch als Herren einer Ortschaft außerhalb des Fayums selbst bezeichnet.

Ihre wirkliche Relevanz gibt diese Art der Zuordnung erst am Ende des ganzen Abschnittes preis. Dort heißt es in einer Beischrift: „Summe der Gaue 6o. Gering/gefallen 6, Summe 66. Ober- und Unterägypten 42, Seeland 24. Summe erneut" (Z. 5or). Ebenso einschlägig ist die monumentale Schlußformel der gesamten Sektion des Papyrus: „Willkommen in Frieden mit seinen Gauen und den Göttern und Göttinnen, die ihre Speise Tag für Tag in ihm haben. Jedes Feuchtgebiet von Ober- und Unterägypten lebt von ihm, und das Gottesland ebenso" (Z. $506-51 \mathrm{II}){ }^{48}$

46 Vgl. Meeks, Mythes et légendes, a. O. (Anm. 5) $225 \mathrm{f}$. und $239 \mathrm{f}$.

47 Der Satz ist von Beinlich, Buch vom Fayum, a. O. (Anm. 8) I6 I m. E. mißverstanden worden, da er den auch Z. I 276 (im Kolophon) bezeugten Priestertitel imì-nčr.i nicht erkannt hat, der mit der griechischen Wiedergabe $\varepsilon \mu \nu \imath \theta \iota$ zu identifizieren ist; vgl. J.F. Quack, „Rezension zu S. Lippert - M. Schentuleit, Demotische Dokumente aus Dime I“, Archiv für Papyrusforschung 53 (2007) 72-73; und zusätzlich Papyrus Tebtunis Tait 22, Z. I, s. M. Depauw, The Demotic Letter. A Study of Epistolographic Scribal Traditions against their Intraand Intercultural Background (2006), S. 351.

48 Auch in Botti A ist mit.t iri zu lesen, Bottis und Beinlichs Umschrift ist zu korrigieren. 
Um diese Aussage ernst zu nehmen, muß man zunächst suchen, wie die im Text real genannten Ortschaften mit den in der Summierung genannten Zahlen korreliert werden können. Das ist gar nicht so einfach. M. E. muß man sich hierfür den gesamten dieser Summierung vorausgehenden Papyrus anschauen, der sich bereits über mehrere Einzelsektionen erstreckt, die auch erkennbar eine Verortung in der Geographie haben bzw. konkreter gesprochen in verschiedenen Bereichen vom Zuflußbereich des Bahr Yussuf und der sonstigen in den See mündenden Wasserläufe bis hin zu einem großen Zentraloval, das den See mit Orten auf seinen Seiten darstellen dürfte.

Im Zuflußbereich haben wir zunächst am oberen Rand 5 definierte Orte, dahinter eine monumentalisierte Inschrift, im unteren 8 Orte sowie Freiraum, in dem ein bis zwei weitere untergebracht werden könnten. In einem eigenen Abschnitt direkt vor der großen Figur der Göttin sind jeweils oben und unten ein weiterer Ort untergebracht, hinter der Göttin ist in quergelegter Schrift der Platz der Achtheit eigens definiert. Im Rahmen des Ovals selbst gibt es oben 20 definierte Orte und noch etwas unbenutzten Freiraum, ebenso im unteren Register, zudem „auf Kante“ quer zum Rest ganz am Ende des Ovals noch einen weiteren Ort. Zusammengenommen macht das also für die vorderen Bereiche 16 definierte Orte, für das Oval 4I. Insgesamt kommt man auf 57, d. h. knapp unterhalb der in der Summe angegebenen Zahl. Ohne dies leider formal absichern zu können, möchte ich die Frage aufwerfen, ob eventuell aufgrund von Überlieferungsproblemen drei Orte, die im selben Umfang wie die vorhandenen hätten dargestellt werden sollen und für die auch Platz vorhanden wäre, versehentlich ausgefallen sind. Damit käme man auf 60, und die als "gering" bzw. "gefallen" angegebenen Orte waren ja logischerweise solche, die keine ausführliche Darstellung erhalten haben. Es scheint mir also zumindest nicht ausgeschlossen, daß die Rechnung früher einmal aufging und tatsächlich 60 Orte mit Bild und Textfeld thematisiert waren, dazu 6 weitere konzeptuell vorhanden, aber aufgrund bestimmter negativer Züge nicht ausgearbeitet. ${ }^{49}$ Die Orte beinhalten einerseits 42 , die als Korrelate zu den einzelnen Gauen Ober- und Unterägyptens betrachtet werden, und andererseits 24 - sozusagen die Umkehrzahl dazu -, die als Spezifika der lokalen Umgebung betrachtet werden.

Zusammengenommen wird hier also ein zweifellos lokales Element entwickelt, das aber Kenntnis und bewußte Anknüpfung an das Gesamtland voraussetzt: Man sieht sich gleichsam als Keimzelle, in der in nuce neben den regional spezifischen Größen über Kultidentifikationen auch das gesamte Land verfügbar ist.

49 Man vergleiche damit, wie im Buch vom Fayum zweimal gesagt wird, bestimmte Dinge würden aus religiöser Scheu nicht schriftlich aufgeführt, s. J.F. Quack, „Explizite Aufzeichnungsmeidung im Alten Ägypten“, Lingua Aegyptia Io (2002) 339-342. 
Wird hiermit also Ägypten in eine einzelne Region und ihre religiöse Topographie hineinprojiziert, so kann man im Buch vom Fayum auch das umgekehrte Phänomen nachweisen, nämlich die Projektion des lokalen Kultes ins ganze Land hinein. Ein Musterbeispiel, wie so etwas geht, stellt die sogenannte Sobeklitanei dar (Z. 590-825).

In ihr werden mit Abbildungen und Beischriften 40 verschiedene Krokodilsgottheiten dargestellt. Jede von ihnen wird durch die Inschrift einem bestimmten ägyptischen Gau zugeordnet, als dessen Gottheit bzw. Herr sie deklariert ist. Dahinter zeigt sich also die durchaus anspruchsvolle Position, daß sich hinter der Maske der unterschiedlichen Lokalgötter aller ägyptischen Gaue doch in tieferer Wahrheit stets der eine Gott verbirgt, nämlich der Krokodilsgott Sobek, Herr des Fayums. Eine solche Hochschätzung des Lokalgottes kann kaum anders als vor Ort entstanden sein. Aber sie setzt voraus, daß die Lokalregion nicht als Lokalregion autonom konzipiert wird, sondern man Wissen um das Land im Ganzen einbringt.

Zusammenfassend kann man also sehen, und könnte es auch noch mit beliebig viel mehr Beispielen hinterfüttern, wie in diesen Handbüchern eine Interaktion zwischen lokaler und überregionaler Theologie stattfindet. Viele Dinge sind ohne Intimkenntnisse vor Ort, insbesondere über geographische Kleinräume und ihre Ressourcen, überhaupt nicht vorstellbar, dürften also tatsächlich als vor Ort entstandene Konzeptionen einzustufen sein. In ihrer konkret überlieferten Ausprägung sind sie aber kaum ohne eine bereits vollzogene Interaktion mit den gesamtägyptischen religiösen Mustern verständlich. Umgekehrt werden auch vor Ort Kenntnisse um Ägypten insgesamt ausgenutzt, um die eigenen religiösen Traditionen dadurch aufzuwerten und zu überhöhen. Wir können also nur von einer gegenseitigen Beeinflussung reden, eine scharfe Trennung von großer und kleiner Tradition ist dagegen kaum möglich. ${ }^{50}$ Als ganz wichtigen Zug möchte ich betonen, wie sich in der Perspektive dieser Texte der Mythos quasi in das Land Ägypten und seine spezifischen Orte eingeschrieben hat, das mit seinen konkreten Erscheinungen und Ressourcen aus den Ereignissen des Mythos resultiert, sei es mit Mineralvorkommen, Fanggebieten, Fauna, Pflanzenbiotopen, oder Orten und Kultformen.

Aber auch wenn wir hier mit lokal entstandenen und allenfalls nachträglich im Sinne der Landesnorm überformten Mythen zu rechnen haben, können wir sie keineswegs ohne weiteres als "Volkstraditionen“ ausgeben. Ein relevanter Punkt,

50 Auch McKim Mariott, in: Village India, a. O. (Anm. 3) I 8 I betont ja die dauernde Interaktion zwischen großer und kleinen Traditionen sowie das Bestehen einer heiligen Literatur, einer Literatenklasse, heiliger Geographie und den damit verbundenen Riten und Zeremonien. 
den man für diese Kompositionen bedenken muß, ist die Frage der Trägerschaft und Verfügbarkeit. Als niedergeschriebene Papyri (bzw. teilweise aus solchen extrahierte Inschriften) sind sie auf Anhieb als Produkt der schriftkundigen Elite zu erkennen. Sofern man ihren Inhalt als Produkt lokaler Volkstraditionen auffassen will, muß man also zum mindesten beachten, daß es sich nicht um ungebrochene Abbilder einer vorgeblich reinen Volkskultur handelt, sondern daß sie durch die Hände von Schreibern und Redakteuren gegangen sind, die mit den landesweiten religiösen Traditionen intim vertraut waren. Die obige Analyse einzelner ausgewählter Passagen hat ja bereits gezeigt, daß sie in ihrer aktuellen Gestalt nur als Ergebnis einer intensiven Interaktion zwischen Lokalgebiet und zentraler Konzeption zu verstehen sind.

Ebenso ist zu beachten, daß es sich teilweise ja nicht einfach um die Sammlung der religiösen Bräuche und Traditionen einer spezifischen Region handelt, sondern um größer angelegte Sammelwerke. Das mythologische Handbuch aus Tebtynis ebenso wie das Handbuch über die Mythologie des Delta sind überregionale Kompilationen. Man kann plausibel annehmen, daß derartige Handbücher als Nachschlagewerke landesweit verfügbar waren und somit überall ein Aufgreifen auch spezifisch lokal angebundener Traditionen leicht möglich war.

Das Buch vom Fayum ist zwar insofern eine lokale Größe, als alle Kopien einigermaßen verläßlich bekannter Herkunft tatsächlich aus dem Fayum stammen, ${ }^{51}$ seine Verfügbarkeit in anderen Regionen läßt sich aber dadurch plausibilisieren, daß Teilbereiche des Textes in einer hieroglyphischen Inschrift im Tempel von Kom Ombo in Oberägypten auftauchen. ${ }^{52}$ Bezugsgröße dürfte hier der in Kom Ombo betriebene Sobekkult gewesen sein, der Verbindungen zum Fayum befördert hat.

Wie sehr die lokalen Ausformungen tatsächlich landesweit greifbar waren, kann man auch an einem anderen Punkt illustrieren. In extremster Verknappung, nämlich einer Reduktion auf Dinge wie die wichtigsten Festdaten, spezifische Bezeichnungen der lokalen männlichen und weiblichen Priester, der heiligen Schlangen, heiligen Bäume, heiligen Seen und heiligen Hügel sowie die Namen vom Ackerland und Sumpfgebiet, gibt es ganz manifest Zusammenstellungen, die überall verfügbar waren. Am besten vertraut ist uns eine derartige Sammlung aus dem sogenannten hieroglyphischen Papyrus von Tanis, zu dem inzwischen ein hieroglyphischer und zwei hieratische Paralleltexte aus Tebtynis hinzugetreten

ऽI Gerade für die besterhaltene hieroglyphische Version, die in Theben angekauft wurde, sind allerdings die Fundumstände in tiefes Dunkel gehüllt, vgl. Beinlich, Buch vom Fayum, a. O. (Anm. 8) is f.

52 J. Yoyotte, „Processions géographiques mentionnant le Fayoum et ses localités“, Bulletin de l'Institut français d'archéologie orientale 61 (1962) 79-138, dort 101; Beinlich, Buch vom Fayum, a. O. (Anm. 8) 64 f. 
sind. ${ }^{53}$ Vor allem kann man sehen, wie im Tempel von Edfu im Soubassement der Außenseite des Sanktuars derartige Angaben ausgezogen und in eine geographische Prozession eingebaut werden (Edfou $\left.\mathrm{I}^{2}, 329-344\right)$. Damit ist eine Zugriffsmöglichkeit über fast ganz Ägypten hin faktisch nachgewiesen; man kann guten Gewissens davon ausgehen, daß diese Sammlung wenigstens im späten Ägypten als kanonisches Wissen an jedem wichtigeren religiösen Zentrum verfügbar war. Tatsächlich ist aus dem „Buch vom Tempel“ sogar konkret nachweisbar, daß der Oberlehrer den Dienstauftrag hatte, den Priesterkindern Kenntnisse im Bereich der spezifischen Lokaltraditionen zu vermitteln. ${ }^{54}$

Tatsächlich griff man sogar in einem noch ganz anderen Ausmaß auf lokale religiöse Traditionen zurück. Es gibt eine ganze Reihe von Kompositionen, in denen die Geographie und die an der Geographie hängenden lokalen kultischen und mythologischen Traditionen ein wesentliches Strukturelement sind. ${ }^{55}$

Zuerst nennen möchte ich dabei eine Passage, die ich bereits kurz erwähnt habe, nämlich den Geierinnentext aus dem sogenannten Thotbuch. In ihm geht es darum, daß der Kandidat für den Zutritt zu den Geheimnissen der Schriftkunst seine Kompetenz auch dadurch nachweist, daß er eine Aufzählung von 42 spezifischen Geierinnen mit ihren Jungen vorlegt. Sie sind jeweils in Situationen und Tätigkeiten beschrieben, welche individuell und spezifisch sind. Dabei hängen sie über die Mittel des Wortspiels, der Ausdeutung des Gauzeichens sowie der Aufnahme lokaler Mythen sehr konkret an den religiösen Traditionen des Gaues, dem sie zugeordnet sind. So wird etwa der Gau von Assiut beschrieben als „Eine Geierin, in deren Hand ihr Junges ist, während es das ausspeit, was es gegessen hat - das ist Assi[ut] ". ${ }^{56}$ Hier wird also das oben behandelte Motiv des speienden Hundes aufgegriffen, aber in der spezifischen Perspektive des Geiertextes umgeschrieben. Diesem geht es darum, die Geierin als Protagonistin des Wissens in ganz Ägypten wiederzufinden, und so setzt er die Geierin eben dort ein, wo die eigentliche

53 F.Ll. Griffith - W.M. F. Petrie, Two Hieroglyphic Papyri from Tanis (1889); J. Osing, Hieratische Texte aus Tebtunis I, The Carlsberg Papyri 2. The Carsten Niebuhr Institute of Near Eastern Studies, Publications 17 (1998) 2I9-275; Osing - Rosati, Papiri geroglifici e ieratici, a.O. (Anm. 4) I9-54. Vgl. zum Inhalt B. H. Stricker, „Aantekeningen of egyptische literatur- en godsdienstgeschiedenis I-II", Oudheidkundige Mededelingen uit het Rijksmuseum van Oudheden te Leiden 25 (I944) 52-82; J. Yoyotte, „La science sacerdotale égyptienne à l'époque gréco-romaine (le papyrus géographique de Tanis)", Bulletin de la société Ernest Renan, NS 9 (1960) I3-I8 = Revue de l'Histoire des Religions I59 (I96I) I33-I38.

54 Quack, in: Beinlich - Hallof - Hussy - von Pfeil (Hgg.), 5. Ägyptologische Tempeltagung, a. O. (Anm. 8) I 65 f.

55 Vgl. J.F. Quack, „Geographie als Struktur in Literatur und Religion“, in: K. Maurer F. Adrom - A. Schlüter (Hgg.), Altägyptische Weltsichten (im Druck).

56 In der Zählung der Erstedition ist diese Stelle Lor (VT), x+2/I 2. 
Lokaltheologie etwas ganz anderes konzipiert hat. Die regionalen religiösen Traditionen werden somit aufgegriffen, aber gerade zur Verherrlichung einer einzigen Konzeption verwendet, der überregionale, landesweite Relevanz gegeben wird.

Besonders häufig ist das Aufgreifen der lokalen religiösen Traditionen im Bereich des Osiris sowie der Funerärkultur. Zumindest in der Spätzeit, für welche man die klarste Dokumentation hat, erhält Osiris einen großen überregionalen Kult, der das Land vereint. Ein gutes Fallbeispiel liefert das sogenannte „Buch vom Durchwandern der Ewigkeit", das in der Römerzeit recht oft als Text dem Verstorbenen mitgegeben wird. ${ }^{57}$ In ihm geht es darum, daß dem Nutznießer im Verklärungsstil gewünscht wird, an den verschiedensten Orten bei den jeweils bedeutenden lokalen Festen zugegen zu sein. Während manche anderen religiösen Kompositionen der Spätzeit dies in relativ klaren Worten ausdrücken, ergeht sich das Buch vom Durchwandeln der Ewigkeit gerade darin, änigmatisch zu sein und seinen Leser zu fordern. Oft wird nur das absolut nötige Minimum an Informationen geboten, mit dem man bei entsprechend guter Bildung eben noch erschließen kann, wann und wo man sich gerade befindet - und selbstverständlich mit dem Kenntnisschatz eines Alten Ägypters, d. h. für heutige Forscher ist es oft kaum noch absicherbar. Bezeichnend ist, daß es durchaus Diskussionen etwa über die korrekte kalendarische Einordnung bestimmter Sektionen gibt. ${ }^{58}$ Damit wird Wissen um die diversen lokalen Einzeltraditionen gleichsam ein definierendes Kriterium für die Zugehörigkeit zu einer intellektuellen Elite.

Noch stärker dürfte der Punkt in den Osirismysterien zu erkennen sein, wie sie besonders in den Osiriskapellen des Tempels von Dendera überliefert sind. Hier werden bewußt die unterschiedlichen Traditionen einzelner Gaue hervorgehoben. Die Hauptkomposition über die Osirismysterien liefert gerade in den ersten Büchern Angaben, die für die wichtigsten Orte des Osiriskultes jeweils spezifische Daten und Verfahren nennt. Das geht bis dahin, daß man etwa in Sais, wie der Text sagt ,abweichend von allem anderen“, die Osirisfigurine aus reiner Erde ohne Getreidebeimischung und ohne Model frei skulptiert und nach Ende der Rituale im Wasser des heiligen Sees deponiert. ${ }^{59}$ Bildlich sieht man auch gut, wie in einer der Kammern von Dendera in schon quasi-antiquarischer Sammelarbeit die Iko-

57 Publikation F.-R. Herbin, Le livre de parcourir l'éternité. Orientalia Lovaniensia Analecta 58 (1994).

58 J.F. Quack, „Rezension zu Fr.-R. Herbin, Le livre de parcourir l'éternité, Orientalistische Literaturzeitung 9I (1996) I 55-I 57; ders., „Geographie als Struktur in Literatur und Religion", im Druck.

59 Vgl. J.F. Quack, „Die rituelle Erneuerung der Osirisfigurinen“, Die Welt des Orients 3 I (2000/or) 5-I 8 , bes. $7 \mathrm{f}$. 
nographie der Osirisformen an verschiedenen Orten nebeneinandergestellt wird (Dendara X, Taf. 25I-259). ${ }^{60}$

Gerade bei Osiris liegt eine Frage auf der Hand, die man vielleicht mit dem plakativen Titel „zergliedern und zusammenfügen" ${ }^{61}$ überschreiben kann. Inwieweit dient die Betonung der lokalen Eigenheiten dazu, Ägypten als disparate Größe erscheinen zu lassen, oder ist ihr Ziel nicht eher, jenseits aller regionalen Spezifika das Vereinende des ganzen Landes zu finden? Der Punkt, an dem man dies am klarsten wiederfinden kann, sind m.E. die Kanopenprozessionen. In ihnen geht es darum, daß aus allen Gauen Ägyptens in einem spezifischen Gefäß Wasser herbeigebracht wird, das konzeptuell als Körperteilreliquie des Osiris im jeweiligen Gau verstanden wird. ${ }^{62}$ Mit dem Wasser wird dann das Getreide begossen, aus dem der neue Kornosiris hergestellt wird. Und so zeigt das Ritual letztlich deutlich, daß die Vielheit der Weg zur Einheit ist. Aus den Einzelteilen aller Regionen ergibt sich ein kohärentes Gesamtprodukt, das wiederum für das Land Frieden und Wohlstand garantieren soll. Dies ist keineswegs ein Einzelfall, sondern der Grundsatz: Wo immer man liebevoll alle einzelnen Lokalbräuche und Mythen zusammenstellt, steht im Hintergrund eben keine partikulare Aussage, sondern eine Botschaft von zentraler, landesweiter Relevanz.

Mehrere Fragen schließen sich sinnvollerweise an diesen Befund an. Zunächst wäre die prinzipielle Frage zu stellen, inwieweit sich hier lokale und zentrale Traditionen gegenseitig beeinflussen. Wie bildet sich überhaupt das, was wir heute als zentrale religiöse Tradition Ägyptens verstehen? Handelt es sich einfach um die ursprünglich lokalen Traditionen der Region, welche politisch dominiert und zur Leitkultur wird? Kandidat wäre hier vor allem die Region um Memphis unter Einschluß von Heliopolis, also der Residenzbereich ab der I. Dynastie. Dies würde erklären, warum gerade die Götterneunheit von Heliopolis und die sich um sie rankenden Mythen so große Bedeutung gewonnen haben. Aber sind die Dinge nicht noch viel komplizierter? Die memphitische Region mag ab der I. Dynastie Hauptregierungssitz und Landeszentrum sein, aber sie ist nicht der alte Traditionsgrund, aus dem die Dynastie stammt. Dies ist vielmehr Oberägypten, wohl spezifischer die Region um Abydos, wo der Friedhof der königlichen Linie noch über viele Generationen bleibt. ${ }^{63}$

60 Vgl. S. Cauville, Dendara, les chapelles osiriennes. Commentaire. Bibliothèque d'étude, Institut français d'archéologie orientale, Kairo I 18 (1997) 199-203.

6I So als Titel des bekannten Aufsatzes von A. Herrmann, „Zergliedern und Zusammenfügen. Religionsgeschichtliches zur Mumifizierung“, Numen 3 (I956) 8I-96.

62 Dendara X, 73-93; vgl. auch H. Beinlich, Die „Osirisreliquien“. Zum Motiv der Körperzergliederung in der altägyptischen Religion. Ägytologische Abhandlungen 42 (1984) 80-289.

63 Vgl. etwa T. Wilkinson, Early Dynastic Egypt (1999) 230-240. 
Zudem kann man ja kaum von der Mythologie der heliopolitanischen Neunheit sprechen, ohne ein Wespennest von Problemen anzustechen. Wir bezeichnen die Neunheit als heliopolitanisch, weil auch die Ägypter selbst sie als Gesamtgröße schon dort verortet haben. Aber es dürfte wenige Forscher geben, welche ernstlich die Theorie vertreten, alle Mitglieder der heliopolitanischen Neunheit seien primär und hauptsächlich in Heliopolis zu Hause. ${ }^{64}$ Isis, Osiris und Horus haben z. B. ausgeprägte Bodenhaftung in den recht nahe beieinander liegenden Regionen von Behbeit el-Hagar, Busiris und Buto im Delta. Nach Heliopolis dürften sie erst sekundär gekommen sein, in dem Maße, wie Re-Atum als verbindlicher Ahn aller Götter festgeschrieben wurde. Das, was wir als zentrale religiöse Konzeptionen Ägyptens verstehen, kann eigentlich erst durch eine spätere Zusammenarbeitung von einst separaten Lokaltraditionen entstanden sein.

Ich möchte aber noch auf einen anderen Punkt fokussieren, nämlich die bewußte Interaktion von Lokalität und Universalismus in den oben angesprochenen Kompositionen. Zum einen ist es vielleicht nicht selbstverständlich, wie sehr offiziell und mit Zustimmung und tatkräftiger Mitarbeit aller staatlichen Stellen hier geradezu der Partikularismus zelebriert wird. Man ist geradezu stolz darauf, daß es so viele unterschiedliche lokale Bräuche um Osiris und so viele ortsgebundene Mythenvarianten gibt, und man macht geradezu ihre korrekte Kenntnis zum markierenden Proprium einer Elite. Kulturpolitik eines Staates muß keineswegs zwangsläufig so aussehen, und man kann sicher aus der Moderne genügend Beispiele aufführen, in denen lokale Traditionen und regionale Sprachen ungern gesehen oder sogar aktiv bekämpft werden. Die Behandlung Tibets durch die Chinesen ist ja gerade wieder ins Auge der Welt getreten, und die Behandlung der Kurden durch die Türken ist, wenngleich derzeit etwas weniger schlagzeilenträchtig, doch auch seit Jahrzehnten virulent.

Nun ist ein wesentlicher Gesichtspunkt für derart unterschiedliche zentrale Reaktionen natürlich in den realen politischen Gegebenheiten zu suchen. Die Kurden wollen lieber heute als morgen einen eigenen Staat, und die Chinesen glauben derzeit wohl nicht, daß ein kulturell autonomes Tibet in allen machtpolitischen Fragen in ihrer Hand bleiben würde. Dagegen war in Ägypten zumindest in der Spätzeit das Risiko eines ernstlichen Separatismus, in dem Sinne, daß etwa das Fayum sich vom Rest des Landes unabhängig erklären würde, völlig irreal. Was immer an kulturellen Divergenzen und direktem Antagonismus der lokalen Regionen zum Zeitpunkt der Reichseinigung auch vorhanden gewesen sein mag, bis zum vollen Einsetzen der von mir ausgewerteten Quellen im I. Jahrtausend v. Chr. gibt es keine ernstlich zentrifugalen Kräften, sondern es wird allenfalls als eine Art von gepflegtem Trachtenverein zelebriert.

64 Vgl. etwa die Diskussion bei H. Kees, Der Götterglaube im alten Ägypten (1956) 254-278. 
Wie wenig für die Macht der Zentrale die divergierenden Lokaltraditionen eine Bedrohung darstellten, kann man vielleicht ganz gut gerade an einem Beispiel illustrieren, das zeigt, bis zu welchen Extremen die Kultivierung einer Ortsidentität in religiösen Fragen gehen konnte. Plutarch, De Iside 72 ( $380 \mathrm{o}$ ) überliefert, daß es in der römischen Zeit Streit zwischen den Gauen von Oxyrhynchos und Kynopolis gab. Die Oxyrhynchiten waren darüber erbost, daß die Kynopoliten den ihnen heiligen Fisch als Speise verzehrten, und aus Rache opferten und verspeisten sie selbst einen Hund, der in Kynopolis heilig war. Die Dinge gerieten außer Kontrolle und die Bewohner der beiden Gaue behandelten einander feindselig und gewaltsam, ${ }^{65}$ bis die römische Macht sie zur Ordnung rief. ${ }^{66}$ Ebenso berichtet Juvenal in einer Satire (15, 27 ff.) (und sicher nicht ohne Übertreibung), daß jede ägyptische Stadt die Götter der anderen haßt, und daß in einer Fehde zwischen Dendera und Kom Ombo sogar ein Mensch getötet und verspeist wurde.

Vielleicht wäre man geneigt, solche Vorkommnisse zunächst als Beleg gegen meine These zu verwenden, beim genaueren Hinschauen zeigt sich aber gerade ihre Stimmigkeit. Plutarch überliefert diese Episode im Zusammenhang seiner Darlegung des ägyptischen Tierkultes nämlich gerade im Rahmen einer Deutung, welche den lokal aufgefächerten Tierkult als bewußte Aufoktroyierung durch den König und den Zentralstaat versteht. Demnach habe der Herrscher Sorge gehabt, die Ägypter könnten als tendenziell aufsässiges Volk, sofern sie nur eine innere Eintracht gewännen, genügend Macht entwickeln, um ihm gefährlich zu sein. Folglich habe er sie angewiesen, verschiedene Tiere zu verehren, und zwar lokal spezifisch jeweils solche, die von Natur aus denen anderer Orte feindlich seien. Ziel ist somit, ein Maß inneren Zwists in der Bevölkerung der verschiedenen Regionen zu schaffen, das es der Zentrale nach dem Prinzip ,divide et impera" leicht macht, die Dinge ungefährdet unter Kontrolle zu halten.

Weiterhin muß man beachten, daß die konzeptuellen geographischen Einheiten Ägyptens, wie sie in der religiösen Topographie hochgehalten werden, herzlich wenig mit den realen Raumstrukturen der Macht zu tun haben. Bezeichnend ist etwa, daß die wichtigste Basistrennung der traditionellen Konzeption Ägyptens die zwischen Ober- und Unterägypten war. Eine solche Organisation ist in der Struktur des Raumes insofern sehr gut nachvollziehbar, als der Kontrast zwischen dem schmalen Alluvialbereich zwischen den Uferbergen einerseits und der weiten Ebene andererseits sehr markant ist. In der politischen Struktur Ägyptens war dies

65 Eine solche Grundeinstellung paßt bestens zum Papyrus Jumilhac, der ja gerade aus dieser Region stammt und von immer neuen Aggressionsversuchen des Seth vom Gau von Oxyrhynchos aus berichtet.

66 Vgl. J. G. Griffiths, Plutarch's De Iside et Osiride (1970) 230-233 und 548 f.; Chr. Froidefond, Plutarque, Euvres morales, tome V, 2e partie. Traité 23. Isis et Osiris (1988; $\left.{ }^{2} 2003\right)$ i $7 \mathrm{f}$. und $24 \mathrm{If}$. 
aber wohl nicht einmal zum Zeitpunkt der sogenannten Reichseinigung ein wirklich markanter Gegensatz - jedenfalls fehlen derzeit klare Beweise dafür, daß es im Delta ein einheitlich organisiertes protostaatliches Gebilde gegeben hat, das als gleichwertige Größe der Einheit entgegentrat, die durch zunehmende Expansionsund Integrationsprozesse in Oberägypten zusammengewachsen war. ${ }^{67}$ Eine reale politische Situation, in der die südliche Deltagrenze eine politische Wasserscheide war, kann es allenfalls sehr ephemer gegeben haben. Man kann gut sehen, wie in allen Epochen schwacher Zentralstaatlichkeit, die es in der ägyptischen Geschichte gegeben hat - die von Forschern heute als Erste, Zweite und Dritte Zwischenzeit bezeichnet werden - die politischen Hauptgrenzen keineswegs zwischen Delta und Oberägypten verlaufen, sondern in Mittelägypten, sozusagen der Region, wo sich die durch Ressourcen und Distanzen bedingte Kontrollfähigkeit von einem nördlichen und einem südlichen Zentrum begegneten. ${ }^{68}$ Und bei einer noch stärkeren Fragmentierung, die es teilweise auch gegeben hat, waren die alten traditionellen Gaugrenzen herzlich irrelevant. Bezeichnend ist, wie in der Ersten Zwischenzeit ein Festungskommandant von Armant im 4. oberägyptischen Gau einen erfolgreichen Militärführer aus dem 3. oberägyptischen Gau um Hilfe bittet, während andere Teile des 4. Gaues sowie der 5. Gau seine Gegner sind. ${ }^{69}$

Hier möchte ich ein abschließendes Fazit ziehen: Es gibt in Ägypten in nicht geringem Maß Mythen und religiöse Konzeptionen, welche regional verortete lokale Entstehungspunkte haben. Sie hängen oft gut erkennbar an den landschaftlichen Ressourcen und topographischen Gegebenheiten, die sie mit einem bis in die Gegenwart der Textautoren reichenden tiefen Sinn erfüllen: Die Götter und ihre Aktionen hinterlassen unauslöschbare Spuren in der Landschaft. Allerdings ist die Entstehung und Ausformulierung dieser Mythen in Gelehrtenkreisen zu suchen, denen auch die landesweite religiöse Tradition gut vertraut war; als Reflexe lokaler Volkstradition kann man sie nur in dem Maße werten, wie solche Volkstraditionen bis in die regionale intellektuelle Elite aufsteigen können. In jedem Fall kann man

67 Vgl. hier etwa T. Wilkinson, „Political Unification: towards a Reconstruction“, Mitteilungen des Deutschen Archäologischen Instituts, Abteilung Kairo 56 (2000) 377-395; B. Kemp, Ancient Egypt. Anatomy of a Civilisation (2006) 73-92.

68 Vgl. hier etwa F. Gomaa, Die libyschen Fürstentümer des Deltas vom Tod Osorkons II. bis zur Wiedervereinigung Ägyptens durch Psametik I. Tübinger Atlas des Vorderen Orients B 6 (1974); ders., Ägypten während der Ersten Zwischenzeit. Tübinger Atlas des Vorderen Orients B 27 (1980); K. A. Kitchen, The Third Intermediate Period in Egypt (1 100-650 BC) (31995); K. Ryholt, The Political Situation in Egypt during the Second Intermediate Period, c. I800-I55o B. C. The Carsten Niebuhr Institute of Near Eastern Studies, Publications 20 (1997).

69 J. Vandier, Mo'alla. La tombe d'Ankhtifi et la tombe de Sébekhotep. Bibliothèque d'étude, Institut français d'archéologie orientale, Kairo I 8 (I950) I $98 \mathrm{f}$. 
keine strikt getrennten "großen“ und „kleinen“ Traditionen postulieren, dafür ist die gegenseitige Interaktion viel zu intensiv, die schon vor Einsetzen aller Quellen stattgefunden haben muß. Die Paradierung der regionalen Unterschiede ist keinesfalls ein Ausdruck eines politischen Partikularismus, sondern immer ein Mittel, die letztliche Einheit des Landes darzustellen.

Prof. Dr. Joachim Friedrich Quack

Ägyptologisches Institut

Universität Heidelberg

Marstallhof 4

D-69II7 Heidelberg

Deutschland

E-Mail: joachim_friedrich.quack@urz.uni-heidelberg.de

\section{Abbildungsnachweis}

Abb. I: nach S. Sauneron, Esna VI/r. Le temple d'Esna Nos 473-546 (1975) 164. 